\title{
Sperm DNA Fragmentation Has a Negative Impact on Cumulative Live Birth Rate of IVF-ET/ICSI in Patients with Oligozoospermia
}

\author{
Liang Xiaodong, Duan Rubing, Ji Peng, Shen Binglian, Mo Ganwen, Guo Jianghua, Liao Yongbin*
}

Reproductive Medicine Center, Jiangmen Central Hospital, Affiliated Jiangmen Hospital of Sun Yat-Sen University, Jiangmen, China

Email address:

229290147@qq.com (Liang Xiaodong),1754930016@qq.com (Duan Rubing),21311732@qq.com (Ji Peng),

404697691@qq.com (Shen Binglian),2507959605@qq.com (Mo Ganwen), 597291523@qq.com (Guo Jianghua),

836944528@qq.com (Liao Yongbin)

*Corresponding author

\section{To cite this article:}

Liang Xiaodong, Duan Rubing, Ji Peng, Shen Binglian, Mo Ganwen, Guo Jianghua, Liao Yongbin. Sperm DNA Fragmentation Has a Negative Impact on Cumulative Live Birth Rate of IVF-ET/ICSI in Patients with Oligozoospermia. Clinical Medicine Research.

Vol. 10, No. 3, 2021, pp. 105-111. doi: 10.11648/j.cmr.20211003.17

Received: May 26, 2021; Accepted: June 17, 2021; Published: June 26, 2021

\begin{abstract}
The relationship between sperm DNA fragmentation and in vitro fertilization (IVF) outcome was uncertain today. In the past, researchers studying the pregnancy outcome of IVF, mainly base on fresh embryo transplantation cycle, recently, cumulative live birth rate was consider as a new indicator of IVF successful rate which is used to evaluate the pregnancy outcome by fresh or thawed transferred embryos until live birth or use up all embryos in one oocyte collection cycle, is more comprehensive. The purpose of this research is to investigate the relationship between sperm DNA fragmentation and cumulative live birth rate. 1019 couples who were assisted by IVF in our center from Jan 2012 to Dec 2016 were studied according to inclusive criteria, and divided into four groups by DNA fragmentation index (DFI), which were determined by sperm chrome structure assay (SCSA), then the fertilization rate, No. of Embryo available and cumulative live birth rate between the groups were analyzed. Cumulative live birth curve was plotted to study the time to reach live birth, and Cox regression model was used in order to find out the factors affecting pregnancy outcome. Study results showed no significant difference in cumulative live birth rate between the four groups, but DFI $\geq 40 \%$ group were significantly lower than the other three groups. In the cumulative live birth curve figure, except the high DFI group, the curve of the other three groups is relatively close. Cox regression model suggest that DFI was not the main factor affecting pregnancy outcome. Further studies in these patients with oligozoospermia showed that when DFI increase, a decrease trend was appeared in cumulative live birth rate, and spend more time to reach live birth studied by the live birth curve. Cox model showed that DFI was the impact factor of pregnancy outcome in oligozoospermia patients. In conclusion, abnormal sperm DNA fragmentation have a negative impact on cumulative live birth rate in patients with oligozoospermia, but further research was still needed.
\end{abstract}

Keywords: Sperm DNA Fragmentation, Cumulative Live Birth Rate, IVF-ET, ICSI, Oligospermia

\section{Introduction}

Sperm DNA fragment is the DNA strand break caused by DNA damage during spermatogenesis under various external factors such as high temperature, radiation, infection and reactive oxygen species. The generation of sperm DNA fragmentation involves the change of genetic material, which can lead to many consequences, such as infertility and recurrent pregnancy loss $[1,2]$. Recent studies have shown that sperm DNA fragments have adverse effects on pregnancy outcomes of assisted reproductive technology [3, 4] and can be predicted for the prognosis of in vitro fertilization (IVF) treatment $[5,6]$. However, previous studies have used clinical pregnancy rate or live birth rate as outcome indicators, mainly based on the fresh embryo transfer cycles. With the rapid development of 
cryopreservation technology, there is a chance for delivery as long as patients still have frozen embryos available. Therefore, these indicators can not access the overall success rate of patients after multiple cycles and the impact of DNA damage on clinical outcomes. Recently, some scholars put forward the concept of cumulative live birth rate, which is used to evaluate the pregnancy outcome of IVF patients who transfer fresh or thawed embryos to live birth or use up all embryos in one oocyte collection cycle [7-9]. As the cumulative live birth rate takes into account factors such as embryo freezing and thawing, the analysis of IVF outcomes is more comprehensive. Therefore, we used it as an outcome index to explore the impact of DNA fragmentation on pregnancy outcomes.

\section{Meterials and Methods}

\subsection{Study Design}

The dataset of IVF patients from January 2012 to December 2016 in our centre were selected, and followed up until December 2018. Inclusion criteria: (1) the age of the female in the first full cycle of fresh cycle is less than or equal to 35 years old; (2) Ovarian stimulation of the female was performed using long Gonadotrophin releasing hormone agonist (GnRH-a) protocol. Exclusion criteria: (1) ovarian reserve function was low, according to Zhang et al. [10]: basic sinus follicle count $<5$, or basic follicle stimulating hormone $(\mathrm{FSH})>10 \mathrm{mmol} / \mathrm{L}$, or once appeared in the previous ovarian stimulation cycle with oocyte retrieval count $<5$; (2) oocyte freezing cycle; (3) follow up from the date of oocyte collection to the end, no live birth record was found, but still have frozen embryos in one oocyte collection cycle.

\subsection{Methods}

\subsubsection{Controlled Ovarian Hyperstimulation and Oocyte Retrieval}

GnRH-a was used in the middle luteal phase according to long GnRH-agonist protocol. 16-20 days after GnRH-a using, recombine FSH was used to start up after patients reach the down-regulating level. At the same time, B-ultrasound was used to monitor the development of follicles and measure the level of serum sex hormone. When the diameter of at least one follicle reached $18 \mathrm{~mm}$ or the diameter of at least three follicles reached $17 \mathrm{~mm}$, human chorionic gonadotrophin (HCG) was injected. The oocytes were retrieved in 34-36 hours after HCG injection.

\subsubsection{Semen Collection and Processing}

After 3-5 days abstinence, semen was collected on the day of oocyte retrieval, and then be processed by the methods of density gradient centrifugation or swim-up.

\subsubsection{In Vitro Fertilization and Embryo Culture}

After oocyte retrieval, oocyte was fertilized by routine IVF protocol. If the fertilization rate is low, rescue intracytoplasm sperm injection (ICSI) program should be used. The embryo was observed on Day 3 after fertilization. High-quality embryo was defined as the embryo with 3-5 cells above grade B of Day 2 or 6-10 cells above grade B of Day 3 . According to the situation of embryo and patients, fresh embryo transfer was carried out, and the remaining embryos were cryopreserved.

\subsubsection{Embryo Thawing and Transferred}

On the 3-5 days of menstruation, the endometrial was monitored and prepared. On the day of transplantation, the frozen tube with embryo was thawed and transferred to the culture medium for transplantation according to frozen embryo transfer protocol. Lacteal support was given after transplantation.

\subsubsection{Sperm Chromatin Structure Assay (SCSA) for Analyzing Sperm DNA Fragmentation Index (DFI)}

After semen liquefaction, Tris-NaCl-EDTA buffer was used for dilution. $0.05 \mathrm{ml}$ diluted suspension was put into flow cytometer tube, $0.1 \mathrm{ml}$ acid treatment solution was added for 30 seconds, and then $0.3 \mathrm{ml}$ acridine orange staining solution was added. The fluorescence of 5000 spermatozoa was detected, and the proportion of all kinds of fluorescence spermatozoa was analyzed by flow cytometer, and DFI was calculated.

\subsubsection{Outcome Measure}

According to the definition of cumulative live birth rate for the complete cycle, select the couples of patients whose embryos have been transferred or who have had live birth records after one oocyte retrieval cycle as the analysis objects. Cumulative live birth rate was defined as women who have at least one live birth per women who achieve one complete cycle.

\subsubsection{Statistical Methods}

For measurement indicators, if the conditions of normal distribution and homogeneity of variance are met, one-way ANOVA is used, if not, nonparametric test is used. Linear correlation trend analysis is used for one-way and orderly classification data. Kaplan Meier method was used to analysis the patients who have multiple oocyte retrieval cycles and plot the cumulative live birth curve, and Cox proportional risk model was constructed for multi factor analysis. Statistical procedure was completed by SPSS 25.0 software, $\mathrm{P}<0.05$ was considered statistically significant.

\section{Results}

\subsection{Patient Characteristic}

Baseline characteristics were shown in Table 1. It can be seen that there is no significant difference in the basic conditions of female patients among the four groups. 
Table 1. Baseline characteristics of female in couples.

\begin{tabular}{llllll}
\hline \multirow{2}{*}{ DFI } & $<\mathbf{2 0 \%}$ & $\mathbf{2 0 \%} \mathbf{3 0} \%$ & $\mathbf{3 0 \%} \mathbf{4 0} \%$ & $>=\mathbf{4 0 \%}$ & P value \\
\cline { 2 - 6 } & $\mathbf{N}=\mathbf{5 8 4}$ & $\mathbf{N}=\mathbf{1 9 0}$ & $\mathbf{N}=\mathbf{1 4 6}$ & $\mathbf{N}=\mathbf{9 9}$ & \\
\hline Female age & $30.29 \pm 3.13$ & $30.03 \pm 3.30$ & $30.27 \pm 3.16$ & $29.89 \pm 3.53$ & 0.585 \\
Body mass index $\left(\mathrm{kg} / \mathrm{m}^{2}\right)$ & $21.69 \pm 3.23$ & $21.91 \pm 3.31$ & $21.27 \pm 3.14$ & $21.00 \pm 2.44$ & 0.112 \\
Duration (years) & $4.12 \pm 2.88$ & $4.21 \pm 2.82$ & $3.68 \pm 2.48$ & $3.67 \pm 2.39$ & 0.249 \\
Primary diagnosis of infertility (\%) & 54.8 & 53.2 & 61.6 & 53.5 & 0.587 \\
Basic FSH (U/L) & $5.54 \pm 1.63$ & $5.63 \pm 1.69$ & $5.88 \pm 2.09$ & $5.65 \pm 1.61$ & 0.254 \\
Basic E2 (pg/ml) & $56.21 \pm 52.41$ & $54.62 \pm 69.24$ & $56.17 \pm 33.50$ & $60.29 \pm 63.75$ & 0.499 \\
No. of oocyte retrieval & $14.62 \pm 6.42$ & $14.19 \pm 6.26$ & $14.81 \pm 6.59$ & $13.29 \pm 5.66$ & 0.282 \\
\hline
\end{tabular}

\subsection{Fertilization and Pregnancy Outcome}

The outcomes of IVF and pregnancy were shown in Table 2. It can be seen from the table that in the first complete cycle, the total fertilization rate of IVF in four groups showed a downward trend with the increase of DFI, while the total cleavage rate of IVF, the total fertilization rate of ICSI, the total cleavage rate of ICSI and the total excellent embryo rate have no significant difference. There was no significant correlation in cumulative live birth rate between the four groups, but through the comparison of the two groups, it was found that the cumulative live birth rate of the DFI $>=40 \%$ group were significantly lower than the other three groups. Figure 1 showed the cumulative live birth curve of the groups in each follow-up month, which showed the time required for the patients to reach live birth. In the figure, except DFI $>=$ $40 \%$ group, the curve of the other three groups is relatively close, which is consistent with the statistical conclusion in Table 2. The common influencing factors were further included in Cox regression model for multivariate analysis [11], and it was found that DFI was not the main factor affecting pregnancy outcome (Table 3 ).

Table 2. Fertilization rate and cumulative live birth rate (in the first complete cycle).

\begin{tabular}{|c|c|c|c|c|c|}
\hline & $<20 \%$ & $20 \% \sim 30 \%$ & $30 \% \sim 40 \%$ & $>=40 \%$ & Pyalue \\
\hline & $N=584$ & $\mathrm{~N}=\mathbf{1 9 0}$ & $N=146$ & $\mathrm{~N}=99$ & I value \\
\hline IVF fertilization rate $(\%)$ & 82.6 & 82.1 & 78.9 & 77.8 & 0.000 \\
\hline IVF cleavage rate (\%) & 98.6 & 98.6 & 98.9 & 99.2 & 0.137 \\
\hline ICSI fertilization rate $(\%)$ & 83.8 & 83.4 & 83.3 & 85.7 & 0.578 \\
\hline ICSI cleavage rate (\%) & 97.4 & 97.7 & 98.3 & 98.1 & 0.276 \\
\hline Good quality embryo rate (\%) & 41.4 & 44.2 & 40.7 & 44.9 & 0.127 \\
\hline No. of Embryo available & $7.36 \pm 4.39$ & $7.49 \pm 4.32$ & $7.52 \pm 4.36$ & $6.93 \pm 4.09$ & 0.649 \\
\hline No. of Embryo transfer & $1.98 \pm 0.26$ & $1.95 \pm 0.26$ & $1.98 \pm 0.28$ & $1.97 \pm 0.26$ & 0.443 \\
\hline Cumulative live birth rate (\%) & 75.7 & 73.7 & 76.7 & 65.7 & 0.137 \\
\hline
\end{tabular}

Table 3. Analysis of cumulative outcome by COX regress model.

\begin{tabular}{|c|c|c|}
\hline & $\mathbf{P}$ & OR \\
\hline Female age & 0.851 & $0.998(0.973-1.023)$ \\
\hline duration & 0.683 & $0.994(0.966-1.023)$ \\
\hline Type of infertility & 0.875 & \\
\hline primary diagnosis of infertility & & 1.000 \\
\hline Secondary infertility & & $0.987(0.844-1.156)$ \\
\hline Basic FSH & 0.593 & $0.987(0.942-1.035)$ \\
\hline Infertility factors & 0.060 & \\
\hline Male & & 1.000 \\
\hline female & 0.009 & $0.774(0.638-0.938)$ \\
\hline both & 0.659 & $0.934(0.689-1.266)$ \\
\hline others & 0.105 & $0.803(0.617-1.047)$ \\
\hline Gn dose & 0.009 & $0.999(0.998-1.000)$ \\
\hline ICSI or not & 0.236 & $1.121(0.928-1.354)$ \\
\hline DFI & 0.335 & $1.303(0.761-2.233)$ \\
\hline PR & 0.288 & $1.001(0.999-1.002)$ \\
\hline Oocyte retrieve count & 0.900 & $0.999(0.988-1.011)$ \\
\hline
\end{tabular}




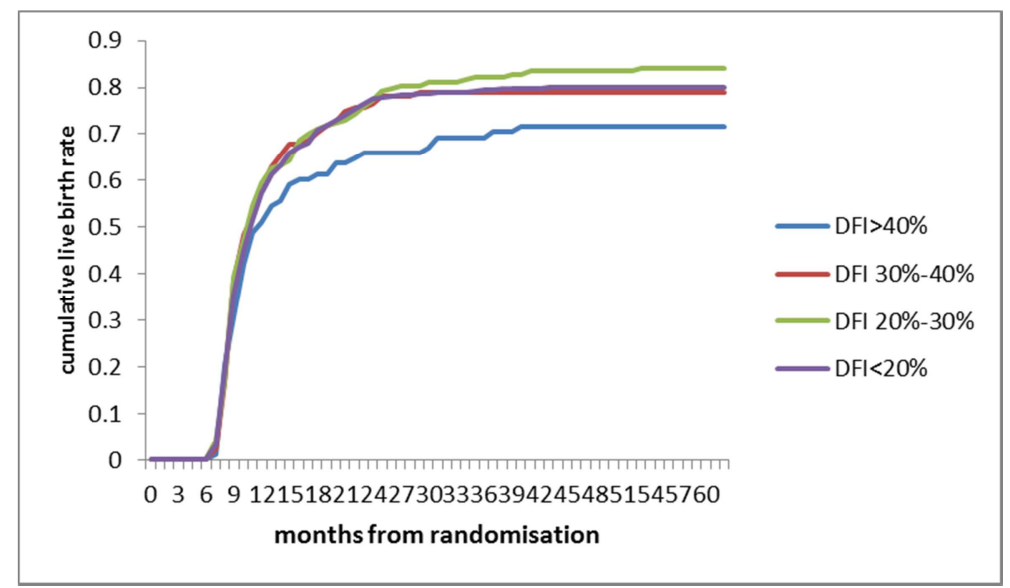

Figure 1. Cumulative live birth curve between the four groups.

\subsection{Analysis of Basic Conditions and Oocyte Number of Couples with Oligozoospermia}

Considering that the total number of sperm is different between patients before semen treatment, the number of sperm with complete DNA is also different, which may be one of the influencing factors. For this reason, we further analyzed the patients whose total sperm in semen was less than $39 \times 10^{6}$. Table 4 showed the basic condition of the female patients with oligozoospermia at the same time. There is no significant difference between the groups.

Table 4. Baseline characteristics of female in couples with oligozoospermia.

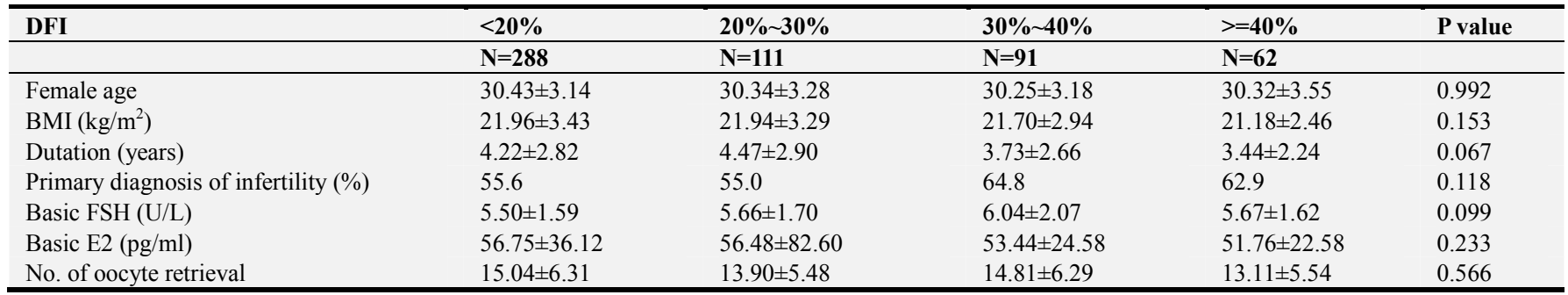

\subsection{Analysis of Pregnancy Outcome of Couples with Oligozoospermia}

The outcomes of in vitro fertilization and pregnancy were shown in table 5. From the perspective of pregnancy outcomes, in the first complete cycle, the cumulative live birth rate of the four groups decreased with the increase of
DFI. The cumulative live birth curve in Figure 2 is significantly different, indicating that couples with high DFI and oligozoospermia need longer time to achieve live birth than those with low DFI. Further multi factor analysis found that DFI was a factor affecting the pregnancy outcome of couples with oligozoospermia (table 6).

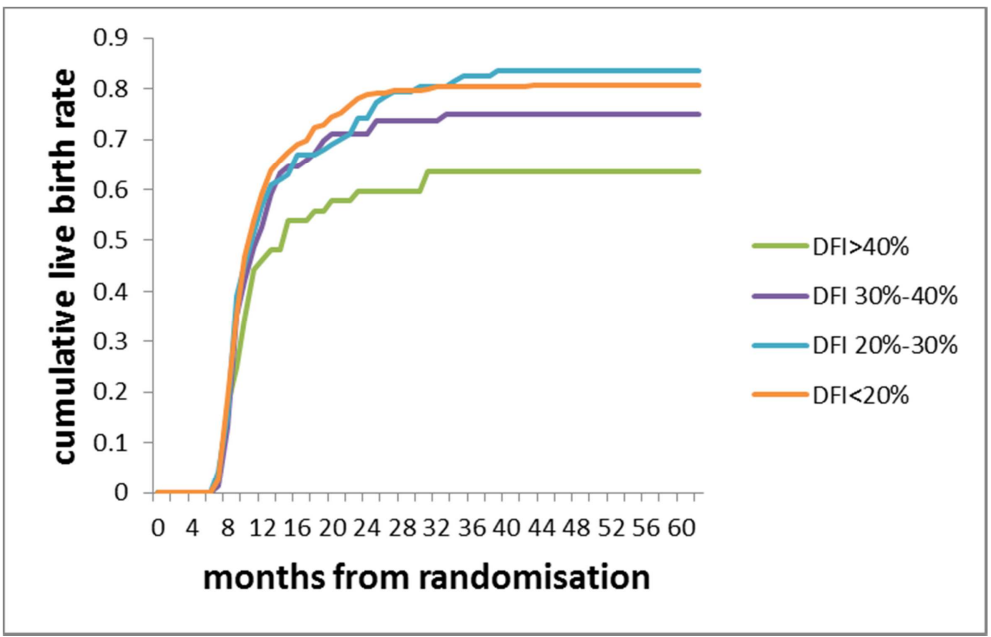

Figure 2. Cumulative live birth curve between the four groups in couples with oligozoospermia. 
Table 5. Fertilization and cumulative outcome in couples with oligozoospermia.

\begin{tabular}{|c|c|c|c|c|c|}
\hline & $<\mathbf{2 0} \%$ & $20 \% \sim 30 \%$ & $30 \% \sim 40 \%$ & $>=40 \%$ & Pvalue \\
\hline & $\mathbf{N}=\mathbf{2 8 8}$ & $\mathrm{N}=111$ & $\mathbf{N}=91$ & $\mathrm{~N}=62$ & \\
\hline IVF fertilization rate $(\%)$ & 79.9 & 78.9 & 74.7 & 75.7 & 0.001 \\
\hline IVF cleavage rate $(\%)$ & 98.3 & 99.1 & 99.1 & 99.1 & 0.059 \\
\hline ICSI fertilization rate $(\%)$ & 83.8 & 83.5 & 82.2 & 84.8 & 0.981 \\
\hline ICSI cleavage rate $(\%)$ & 97.8 & 97.2 & 98.6 & 97.9 & 0.606 \\
\hline Good quality embryo rate $(\%)$ & 43.7 & 45.9 & 40.3 & 43.4 & 0.327 \\
\hline No. of Embryo available & $7.80 \pm 4.48$ & $7.37 \pm 3.91$ & $7.37 \pm 3.98$ & $6.69 \pm 4.04$ & 0.309 \\
\hline Cumulative live birth rate & 77.4 & 72.1 & 71.4 & 59.7 & 0.006 \\
\hline
\end{tabular}

Table 6. Analysis of cumulative outcome by COX regress model in couples with oligozoospermia.

\begin{tabular}{|c|c|c|}
\hline & $\mathbf{P}$ & OR \\
\hline Female age & 0.928 & $0.998(0.964-1.034)$ \\
\hline duration & 0.531 & $1.013(0.973-1.054)$ \\
\hline Type of infertility & 0.796 & \\
\hline primary diagnosis of infertility & & 1.000 \\
\hline Secondary infertility & & $1.029(0.826-1.283)$ \\
\hline Basic FSH & 0.235 & $0.962(0.902-1.026)$ \\
\hline Infertility factors & 0.106 & \\
\hline Male & & 1.000 \\
\hline female & 0.020 & $0.743(0.579-0.954)$ \\
\hline both & 0.445 & $0.861(0.588-1.263)$ \\
\hline others & 0.124 & $0.740(0.504-1.086)$ \\
\hline Gn dose & 0.031 & $0.999(0.998-1.000)$ \\
\hline ICSI or not & 0.096 & $1.221(0.965-1.545)$ \\
\hline DFI & 0.017 & $2.387(1.167-4.880)$ \\
\hline PR & 0.467 & $1.003(0.995-1.010)$ \\
\hline Oocyte retrieve count & 0.432 & $1.007(0.990-1.024)$ \\
\hline
\end{tabular}

\section{Discussion}

With the development of embryo freezing technology, patients who fail to succeed in IVF fresh embryo transfer cycle can freeze the available surplus embryos, so as to thaw the embryos for transfer under suitable conditions in the future and improve the probability of success, especially for patients who are not suitable for embryo transfer due to various special reasons in the fresh cycle. It is a strategy to protect the safety of patients and ensure the chance of pregnancy. However, so far, in the related research on the effect of sperm DNA fragments on pregnancy outcome, the evaluation method is still mainly based on fresh embryo transfer cycle, and the indicators of clinical pregnancy and live birth mainly refer to the pregnancy rate and live birth rate of fresh cycle. Only a few reports analyze the effect of sperm DNA fragments from the perspective of thawing transfer cycle [12]. Cumulative live birth rate refers to the live birth rate of the embryo obtained by one oocyte retrieval until delivery is completed or no embryo can be transferred. It defines all transfer cycles subordinate to the oocyte retrieval cycle as a complete cycle, including both fresh embryo transfer and thawing transfer cycle [9]. In addition, the time from oocyte retrieval to live birth can be calculated and the cumulative live birth curve can be plotted. Based on the above advantages, the cumulative live birth rate has been recognized and used by more and more scholars [13, 14], and it is more appropriate to evaluate the impact of sperm DNA fragments.
In this study, excluding ovarian function and basic conditions, sperm DNA fragmentation had no significant effect on the cumulative live birth rate in the first complete cycle, and the cumulative live birth curve in each follow-up month was also relatively close, indicating that sperm DNA fragmentation has multiple effects on pregnancy outcome. The first effect taken into consideration may be that the sperm sample tested by SCSA comes from crude semen, while sperm used in IVF/ICSI program have been processed [12]. Moreover, the embryos obtained by IVF or ICSI have been screened before transplantation, no matter in the fresh or thawed transfer cycle, so that good embryos can be left for transplantation. Finally, the endometrial receptivity on the day of transplantation needs to be considered.

Considering that the total number of sperm among patients before semen treatment is different, the absolute number of sperm is also different although the same DNA fragments rate, which may be one of the influencing factors, so we further study the impact of sperm DNA fragmentation on outcome with oligospermia. In this study, patients with high DNA fragmentation rate began to show a certain impact on the cumulative pregnancy outcome, indicating that although these patients obtained fertilization mainly through ICSI technology, they still failed to change the poor outcome [15, $\mathrm{x} 16]$. As oligozoospermia itself can also affect pregnancy outcome, Cox multivariate regression analysis model was used in this study to find out other associated risk factors and independent factors affecting pregnancy outcome. The results showed that sperm DNA fragmentation was an independent factor in oligozoospermia patients, suggesting that high DNA 
fragmentation rate would seriously affect the fertilization rate and pregnancy outcome in patients with oligozoospermia. Low sperm number and high DNA fragmentation rate mean that the absolute number of sperm with normal DNA is also relatively small, which reduces the fertilization rate and further affects the cumulative live birth rate. Mehta et al. found that for patients with severe oligozoospermia, using testicular sperm has higher pregnancy rate and lower ICSI abortion rate than ejaculating sperm, because testicular sperm has more sperm with complete DNA than ejaculating sperm, which showed that DNA fragmentation is of more important clinical significance in patients with oligozoospermia [17]. In patients with a large number of sperm, although the rate of DNA fragments is high, there will still be a certain number of normal sperm. After in vitro semen treatment, the number of sperm may still meet the needs of in vitro fertilization. This is the reason why WHO manual emphasizes the principle that the total number of sperm is priority.

In addition, this study also found that DNA fragmentation rate had a more significant effect on IVF fertilization rate, but had little effect on ICSI total fertilization rate, indicating that most of these patients obtained fertilization through rescue ICSI technology, and ultimately improved embryo quality and pregnancy outcome. This suggests that sperm DNA fragmentation mainly affects the natural process of sperm oocyte combination [18]. In the past, the fertilization rate of IVF was low, and the reason was usually found from acrosin, acrosome reaction or oocyte maturity. Recent studies have shown that the fragmentation rate of sperm nuclear DNA can also affect the combination of sperm and oocyte [19-22] However, the DNA damage of sperm occurs in the nucleus of sperm, while the recognition between sperm and oocyte only occurs on the surface of both. When sperm contacts with oocyte, it is sperm membrane rather than sperm nucleus and oocyte membrane that adheres and recognizes. This suggests that sperm DNA damage may affect the process of recognition with oocytes. However, due to the multiple types of sperm DNA damage [23], the DNA repair during spermatogenesis [24, 25] and the mechanism of interaction with oocytes are more complex [26], further research is needed to reveal the relationship between sperm DNA damage and the process of recognition with oocytes.

\section{Conclusion}

This study is the first report to study the relationship between sperm DNA fragmentation and cumulative live birth rate. It is found that high DNA fragmentation rate has a significant impact on the total number of sperm, but many factors still need to be considered for in-depth analysis. Secondly, DNA fragments may have a certain predictive value for IVF fertilization. Whether DNA fragments can be used as a predictive indicator for the selection of fertilization methods, as well as its mechanism and influence, still need further research in-depth and multi center.

\section{References}

[1] Haddock L., Gordon S., Lewis SEM., Larsen P., Shehata A., Shehata H.(2021). Sperm DNA fragmentation is a novel biomarker for early pregnancy loss. Reproductive BioMedicine Online, 42 (1), 175-184.

[2] Santi D., Spaggiari G., Simoni M. (2018). Sperm DNA fragmentation index as a promising predictive tool for male infertility diagnosis and treatment management - metaanalyses. Reproductive BioMedicine Online, 37 (3), 315-326.

[3] Nicopoullos J., Vicens-Morton A., Lewis SEM., Lee K., Larsen P., Ramsay J., Yap T., Minhas S.(2019). Novel use of COMET parameters of sperm DNA damage may increase its utility to diagnose male infertility and predict live births following both IVF and ICSI. HUMAN REPRODUCTION, 4 (10), 1915-1923.

[4] Deng C., Li T., Xie Y., Guo Y., Yang Q. Y., Liang X., Deng C. H., Liu G. H. (2019). Sperm DNA fragmentation index influences assisted reproductive technology outcome: A systematic review and meta-analysis combined with a retrospective cohort study. Andrologia, 51 (6), e13263.

[5] Cissen M., Wely M. V., Scholten I., Mansell S., Bruin J. P., Mol B. W., Braat D., Repping S., Hamer G. (2016). Measuring Sperm DNA Fragmentation and Clinical Outcomes of Medically Assisted Reproduction: A Systematic Review and Meta-Analysis. PLoS One, 11 (11), e 0165125.

[6] Tang L., Rao M., Yang W., Yao Y., Luo Q., Lu L., Wang L., Zhao S. (2021). Predictive value of the sperm DNA fragmentation index for low or failed IVF fertilization in men with mild-to-moderate asthenozoospermia. Journal of Gynecology Obstetrics and Human Reproduction, 50 (6), 101868 .

[7] McLernon D. J., Maheshwari A., Lee A. J., Bhattacharya S. (2016). Cumulative live birth rates after one or more complete cycles of IVF: a population-based study of linked cycle data from 178, 898 women. HUMAN REPRODUCTION, 31 (3), $572-581$.

[8] Toftager M., Bogstad J., Lossl K., Praetorius L., Zedeler A., Bryndorf T., Nilas L., Pinborg A. (2017). Cumulative live birth rates after one ART cycle including all subsequent frozen-thaw cycles in 1050 women: secondary outcome of an RCT comparing GnRH-antagonist and GnRH-agonist protocols. HUMAN REPRODUCTION, 32 (3), 556-567.

[9] Maheshwari A., McLernon D., Bhattacharya S. (2015). Cumulative live birth rate: time for a consensus? HUMAN REPRODUCTION, 30 (12), 2703-2707.

[10] Zhang H. H., Xu P. Y., Wu J., Zou W. W., Xu X. M., Cao X. Y., Wei L. Z. (2014). Dehydroepiandrosterone improves follicular fluid bone morphogenetic protein-15 and accumulated embryo score of infertility patients with diminished ovarian reserve undergoing in vitro fertilization: a randomized controlled trial. Journal of Ovarian Research, 7, 93.

[11] Missmer S. A., Pearson K. R., Ryan L. M., Meeker J. D., Cramer D. W., Hauser R. (2011). Analysis of multiple-cycle data from couples undergoing in vitro fertilization: methodologic issues and statistical approaches. EPIDEMIOLOGY, 22 (4), 497-504. 
[12] Ni W., Xiao S., Qiu X., Jin J., Pan C., Li Y., Fei Q., Yang X., Zhang L., Huang X. (2014). Effect of sperm DNA fragmentation on clinical outcome of frozen-thawed embryo transfer and on blastocyst formation. PLoS One, 9 (4), e94956.

[13] Wu C. X., Zhang T., Shu L., Huang J., Diao F. Y., Ding W., Gao Y., Wang W., Mao Y. D., Cui Y. G., Liu J. Y. (2018). Cumulative live birth rates per oocytes retrieved cycle: evaluation of clinical outcomes of IVF/ICSI. Zhonghua Fu Chan Ke Za Zhi, 53 (3), 160-166.

[14] Zhou J., Wang B., Hu Y., Sun H. (2017). Association between the number of oocytes retrieved and cumulative live birth rate in women aged 35-40 years undergoing long GnRH agonist IVF/ICSI cycles. ARCHIVES OF GYNECOLOGY AND OBSTETRICS, 296 (5), 1005-1012.

[15] Mehta A., Bolyakov A., Schlegel P. N., Paduch D. A. (2015). Higher pregnancy rates using testicular sperm in men with severe oligospermia. FERTILITY AND STERILITY, 104 (6), 1382-1387.

[16] Zini A. (2015). Use of testicular sperm for intracytoplasmic sperm injection in oligozoospermic couples with high levels of sperm deoxyribonucleic acid fragmentation. FERTILITY AND STERILITY, 104 (6), 1376-1377.

[17] Esteves S. C., Sanchez-Martin F., Sanchez-Martin P., Schneider D. T., Gosalvez J. (2015). Comparison of reproductive outcome in oligozoospermic men with high sperm DNA fragmentation undergoing intracytoplasmic sperm injection with ejaculated and testicular sperm. FERTILITY AND STERILITY, 104 (6), 1398-1405.

[18] Oleszczuk K., Giwercman A., Bungum M. (2016). Sperm chromatin structure assay in prediction of in vitro fertilization outcome. Andrology, 4 (2), 290-296.
[19] Bach P. V., Schlegel P. N. (2016). Sperm DNA damage and its role in IVF and ICSI. Basic Clin Androl, 26, 15-24.

[20] Pregl Breznik B., Kovacic B., Vlaisavljevic V. (2013). Are sperm DNA fragmentation, hyperactivation, and hyaluronanbinding ability predictive for fertilization and embryo development in in vitro fertilization and intracytoplasmic sperm injection? FERTILITY AND STERILITY, 99 (5), 1233 1241.

[21] Jiang W. J., Jin F., Zhou L. M. (2016). Values of the sperm deformity index, acrosome abnormity rate, and sperm DNA fragmentation index of optimized sperm in predicting IVF fertilization failure. Zhonghua Nan Ke Xue, 22 (2), 147-152.

[22] Parmegiani L., Cognigni G. E., Filicori M. (2014). Sperm selection: effect on sperm DNA quality. Advances in Experimental Medicine and Biology, 791, 151-172.

[23] Wei R. X., Chen J. W., Huang J. H., Zhang X. X., Cui Y. (2015). Type of sperm DNA strand breaks in infertile men and its clinical implication. Zhonghua Nan Ke Xue, 21 (7), 604609 .

[24] Xu P., Cai X., Zhang W., Li Y., Qiu P., Lu D., He X. (2016). Flavonoids of Rosa roxburghii Tratt exhibit radioprotection and anti-apoptosis properties via the $\mathrm{Bcl}-2(\mathrm{Ca}(2+)) / \mathrm{Caspase}-$ 3/PARP-1 pathway. APOPTOSIS, 21 (10), 1125-1143.

[25] Zhang G. W., Cai H. C., Shang X. J. (2016). Ubiquitinproteasome system and sperm DNA repair: An update. Zhonghua Nan Ke Xue, 22 (9), 834-837.

[26] Jin J., Pan C., Fei Q., Ni W., Yang X., Zhang L., Huang X. (2015). Effect of sperm DNA fragmentation on the clinical outcomes for in vitro fertilization and intracytoplasmic sperm injection in women with different ovarian reserves. FERTILITY AND STERILITY, 103 (4), 910-916. 